Pacific Journal of Mathematics

DERIVATIONS WITH INVERTIBLE VALUES IN RINGS WITH 


\title{
DERIVATIONS WITH INVERTIBLE VALUES IN RINGS WITH INVOLUTION
}

\author{
A. Giambruno, P. Misso and C. Polcino Milies
}

Let $R$ be a semiprime 2-torsion free ring with involution * and let $S=\left\{x \in R \mid x=x^{*}\right\}$ be the set of symmetric elements. We prove that if $R$ has a derivation $d$, non-zero on $S$, such that for all $s \in S$ either $d(s)=0$ or $d(s)$ is invertible, then $R$ must be one of the following: (1) a division ring, (2) $2 \times 2$ matrices over a division ring, (3) the direct sum of a division ring and its opposite with exchange involution, (4) the direct sum of $2 \times 2$ matrices over a division ring and its opposite with exchange involution, (5) $4 \times 4$ matrices over a field with symplectic involution.

Recently Bergen, Herstein and Lanski studied the structure of a ring $R$ with a derivation $d \neq 0$ such that, for each $x \in R, d(x)=0$ or $d(x)$ is invertible. They proved that, except for a special case which occurs when $2 R=0$, such a ring must be either a division ring $D$ or the ring $D_{2}$ of $2 \times 2$ matrices over a division ring.

In this paper we address ourselves to a similar problem in the setting of rings with involution, namely: let $R$ be a 2-torsion free semiprime ring with involution and let $S$ be the set of symmetric elements. If $d \neq 0$ is a derivation of $R$ such that the non-zero elements of $d(S)$ are invertible, what can we conclude about $R$ ?

We shall prove that $R$ must be rather special. In fact we shall show the following:

THEOREM. Let $R$ be a 2-torsion free semiprime ring with involution. Let $d$ be a derivation of $R$ such that $d(S) \neq 0$ and the non-zero elements of $d(S)$ are invertible in $R$. Then $R$ is either:

1. a division ring $D$, or

2. $D_{2}$, the ring of $2 \times 2$ matrices over $D$, or

3. $D \oplus D^{\mathrm{op}}$, the direct sum of a division ring and its opposite relative to the exchange involution, or

4. $D_{2} \oplus D_{2}^{\text {op }}$ with the exchange involution, or

5. $F_{4}$, the ring of $4 \times 4$ matrices over a field $F$ with symplectic involution.

In case $R=F_{4}$ with ${ }^{*}$ symplectic we shall prove that $d$ is inner. As Herstein has pointed out, an easy example of such a ring is given by 
taking $F$ to be a field in which -1 is not a square and $d$ the inner derivation in $F_{4}$ induced by $\left(\begin{array}{cc}0 & I \\ -1 & 0\end{array}\right)$ where $I$ is the identity matrix in $F_{2}$.

Now, if $R=D \oplus D^{\text {op }}$ or $R=D_{2} \oplus D_{2}^{\text {op }}$ then $S \cong D$ or $S \cong D_{2}$ respectively. Thus both cases come naturally from [1].

We remark that if $d(S)=0$ then $d(\bar{S})=0$, where $\bar{S}$ is the subring generated by $S$; hence, if $R$ is semiprime, by [3, Theorem 2.1.5] either $S$ lies in the center of $R$ (and $R$ satisfies the standard identity of degree 4) or $d(J)=0$ for some non-zero ideal $J$ of $R$.

Let $R$ be a ring with involution; we denote by $Z$ the center of $R$ and by $S$ and $K$ the sets of symmetric and skew elements of $R$ respectively. Throughout this paper, unless otherwise stated, $R$ will be a 2-torsion free semiprime ring with an involution * and $d$ will be a derivation of $R$ such that $d(S) \neq 0$ and the non-zero elements of $d(S)$ are invertible.

We begin with the following

LEMMA 1. If $I=I^{*}$ is a non-zero ideal of $R$ then $d(I \cap S) \neq 0$.

Proof. Suppose, by contradiction, that $d(I \cap S)=0$ and let $t \in S$ be such that $d(t) \neq 0$. For all $S \in I \cap S$ the elements sts and $s t+t s$ lie in $I \cap S$, hence

$$
\begin{aligned}
& 0=d(s t s)=s d(t) s \\
& 0=d(s t+t s)=s d(t)+d(t) s
\end{aligned}
$$

Multiplying the second equality from the left by $s$, we obtain $s^{2} d(t)=0$. Now, from our basic hypothesis on $R, d(t)$ is invertible; hence $s^{2}=0$, for all $s \in I \cap S$.

Now let $x \in R, s \in I \cap S$. Then the element $s x+x^{*} s$ lies in $I \cap S$ and, so, it must be square-zero. Therefore, since $s^{2}=0$,

$$
0=\left(s x+x^{*} s\right) s x=(s x)^{3},
$$

that is, every element in the right ideal $s R$ is nilpotent of index $\leq 3$. By Levitski's Theorem [2, Lemma 1] we must have $s R=0$ and, so, $s=0$. This proves that $I \cap S=0$.

For $x \in I, x+x^{*} \in I \cap S$; hence $x=-x^{*}$ and $x^{2} \in I \cap S=0$. This $I$ is a nilideal of index $\leq 2$. This forces $I=0$, a contradiction.

At this stage we are able to prove our result in case $R$ is not simple; in fact we have

Proposition 1. If $R$ is not a simple ring then either $R \cong D \oplus D^{\mathrm{op}}, D$ a division ring, or $R \cong D_{2} \oplus D_{2}^{\mathrm{op}}$ and $*$ is the exchange involution. 
Proof. Let $I \neq R$ be an ideal of $R$ such that $I=I^{*}$.

Since $d\left(I^{2} \cap S\right) \subset d\left(I^{2}\right) \subset I$, Lemma 1 shows that $I^{2}=0$ and the semiprimeness of $R$ forces $I=0$. We have proved that $R$ does not contain proper ${ }^{*}$-ideals.

If $R$ is not simple, then there exists a proper ideal $I \neq I^{*}$. Since $I+I^{*}$ is a non-zero ${ }^{*}$-ideal of $R, I+I^{*}=R$. Also $I \cap I^{*} \neq R$ is a *-ideal of $R$, hence $I \cap I^{*}=0$. Thus we have that $R=I \oplus I^{*}$. Moreover since $I^{2} \neq I^{* 2}$ we also get $R=I^{2} \oplus I^{* 2}$ and, so, $I=I^{2}$ and $I^{*}=I^{* 2}$; hence, they are both invariant under $d$. Clearly $S=\left\{x+x^{*}\right\}$ $x \in I\}$ and so $d(x)$ and $d\left(x^{*}\right)$ are both 0 or both units in $I$ and $I^{*}$ respectively.

By [1, Theorem 1] $I$, and hence also $I^{*}$, is either a division ring $D$ or $D_{2}$. If $d(I)=0$, then $d\left(I^{*}\right) \neq 0$ and the argument above leads to the same conclusion. Clearly the involution in $R$ is the exchange involution.

If $R$ is a prime ring we denote by $C$ the extended centroid of $R$ and by $Q=R C$ the central closure of $R$ (see [3, pg. 22]). The next lemma holds for arbitrary rings with involution, with a derivation $d \neq 0$.

LEMMA 2. Let $R$ be a prime ring with involution, with a derivation $d \neq 0$. Let $x \in R$ be such that for all $s \in S$

$$
x s x^{*} d(R) x s x^{*}=0 .
$$

Then either $x^{*} d(R) x=0$ or $Q=R C$ has a minimal right ideal.

Proof. For $y \in R$ let $u=x^{*} d(y) x$. Then if $s \in S$, ususu $=u s u s u^{*}=$ 0 ; now, if $r \in R, s u^{*}{ }^{*}+r u s \in S$ and, so,

$$
0=v s u\left(s u^{*} r^{*}+r u s\right) u\left(s u^{*} r^{*}+r u s\right) u=u s u r u s u r u s u .
$$

This says that every element in the right ideal $u s u R$ is nilpotent of index $\leq 3$. By Levitski's theorem [2, Lemma 1.1], $u s u R=0$ and so $u s u=0$ for all $s \in S$. By [5, Lemma 3], if $u \neq 0, Q=R C$ has a minimal right ideal.

In light of Proposition 1 we now make a first reduction: from now on, unless otherwise stated, we will always assume that $R$ is a simple ring with 1 . In this case clearly $R$ coincides with its own central closure.

The next lemmas give us some information about the nature of the symmetric elements in the kernel of $d$.

Lemma 3. Let $a \in S$. If for all $s \in S$ we have that asa $=\lambda a$, for some $\lambda=\lambda(s) \in z$, then $R$ has a minimal right ideal. 
Proof. Let $x \in R$. Then $a\left(x+x^{*}\right) a=\lambda a$, for some $\lambda \in Z$, that is $a x^{*} a=\lambda a-a x a$. Let $\mu \in Z$ be such that $a\left(x a x+x^{*} a x^{*}\right) a=\mu a$. Playing these off against each other we get

$$
0=\operatorname{axaxa}+a x^{*} a x^{*} a-\mu a=2 \operatorname{axaxa}-2 \lambda a x a+\left(\lambda^{2}-\mu\right) a .
$$

Therefore $2(a x)^{3}-2 \lambda(a x)^{2}+\left(\lambda^{2}-\mu\right) a x=0$ and, since char $R \neq 2$, ax is algebraic over $Z$ of degree at most 3. This proves that $a R$ is an algebraic algebra of bounded degree. Thus $a R$ satisfies a polynomial identity; hence $R$ satisfies a generalized polynomial identity. Since $R$ coincides with its own central closure, by a theorem of Martindale [3, Theorem 1.3.2.] $R$ has a minimal right ideal.

Lemma 4. Suppose $R$ does not contain minimal right ideals. If $a \in S$ is such that $d(a)=0$ then either $a$ is invertible or $a d(R) a=0$.

Proof. Suppose $a \neq 0$ and $a$ is not invertible. Since $d(a)=0$ then, for all $s \in S, d(a s a)=a d(s) a$ and it is not invertible. Hence $a d(s) a=0$.

Now let $x \in R$. Then $a d\left(x+x^{*}\right) a=0$ implies $a d(x) a=-a d\left(x^{*}\right) a$. Therefore for all $s \in S$, recalling that $d(a)=a d(s) a=0$ we get

$$
\operatorname{asad}(x) a=\operatorname{ad}(\operatorname{sax}) a=-a d\left(x^{*} \text { as }\right) a=-a d\left(x^{*}\right) \text { asa }=\text { ad }(x) \text { asa. }
$$

We have proved that for all $x \in R, s \in S$,

$$
\text { asad }(x) a=a d(x) \text { asa }
$$

Since $d(a)=0, d(a R) \subset a R$; moreover if $\rho_{R}(a)$ is the left annihilator of $a$ in $R, d\left(\rho_{R}(a)\right) \subset \rho_{R}(a)$; this says that $d$ induces a derivation (which we will still denote by $d$ ) in the prime ring $R_{1}=a R / \rho_{R}(a) \cap a R$. Moreover, for $s \in S$, if $\overline{a s}$ is the image of as in $R_{1}$, from (1) we get

$$
\overline{a s} d(\overline{a x})=d(\overline{a x}) \overline{a s}, \quad \text { for all } \overline{a x} \in R_{1} \text {. }
$$

By [4] since char $R \neq 2$ either $d=0$ in $R_{1}$ or $\overline{a s} \in Z\left(R_{1}\right)$, the center of $R_{1}$. That is, either $a d(R) a=0$ or asaxa= axasa for all $x \in R$.

If $\operatorname{ad}(R) a=0$ we are done; therefore we may assume that asaxa= axasa, for all $x \in R, s \in S$. But then, by [3, Lemma 1.3.2.], as $a=\lambda a$, for some $\lambda \in Z$ and, by Lemma 3, $R$ has a minimal right ideal, a contradiction.

We remark that since $R$ is simple with 1 then it must be a primitive ring. Now, through a repeated application of the density theorem we will be able to prove that $R$ is artinian. 
Proposition 2. $R$ is a simple artinian ring.

Proof. Since $R$ is primitive it is a dense ring of linear transformations on a vector space $V$ over a division ring $D$. By [3, Lemma 1.1.2.] to prove that $R$ is artinian it is enough to prove that $R$ has a minimal right ideal or equivalently that $R$ contains a non-zero transformation of finite rank. Suppose, by contradiction, that this is not the case.

Let $s \in S$ be such that $d(s) \neq 0$ and suppose that there exist linearly independent vectors $v, w \in V$ such that

$$
v s=w s=0 .
$$

Since $d(s)$ is invertible, the vectors $v d(s)$ and $w d(s)$ are linearly independent over $D$. Moreover, since $R$ doesn't contain non-zero transformations of finite rank, there exists a vector $u \in V$ such that $u s \notin v d(s) D+$ $w d(s) D$, i.e., $u s, v d(s), w d(s)$ are linearly independent over $D$.

By the density of the action of $R$ on $V$, there exists $x \in R$ such that

$$
\begin{array}{r}
u s x \neq 0 \\
v d(s) x=0 \\
w d(s) x \neq 0 .
\end{array}
$$

Let $t \in S$. Since $v d(s) x=v s=0$ then $v d\left(\operatorname{sxtx} *_{s}\right)=0$; hence, since $s x t x^{*} \in S$ and $d\left(s x t x^{*} s\right)$ is not invertible, we must have $d(s x t x * s)=0$. Moreover $s$, and so $s x t x^{*} s$, is not invertible. Since $R$ has no minimal right ideals, by applying Lemma 4 to the element sxtx*s, we get $s x t x * s d(R) s x t x * s=0$, for all $t \in S$. Hence Lemma 2 implies $x * s d(R) s x$ $=0$.

Now let $y, z \in R$. Since $x^{*} s d(y) s x=0$ we have

$$
0=x^{*} s d(y s x z) s x=x^{*} s y d(s x z) s x .
$$

Hence $x^{*} s R d(s x R) s x=0$ and, since $x^{*} s \neq 0$, the primeness of $R$ forces $d(s x R) s x=0$. If $y \in R$ we get

$$
0=d(s x y) s x=d(s) x y s x+s d(x y) s x ;
$$

hence, since $w s=0,0=w d(s x y) s x=w d(s) x y s x$. But $w d(s) x \neq 0$, and, by the density of the action of $R$ on $V, w d(s) x R=V$; thus $0=$ $w d(s) x R s x=V s x$ implying $s x=0$, a contradiction.

We have proved that for every $s \in S$ with $d(s) \neq 0, \operatorname{dim}_{D} \operatorname{ker} s \leq 1$.

Now let $W$ be a finite dimensional subspace of $V$ such that $\operatorname{dim}_{D} W$ $>1$ and let $\rho=\rho_{w}=\{x \in R \mid W x=0\} ; \rho$ is a right ideal of $R$. 
We claim that there exists $s \in \rho \cap S$ such that $s^{2} \neq 0$. In fact, suppose not and let $x \in \rho, s \in \rho \cap S$. Then, since $\left(x s+s x^{*}\right) \in \rho \cap S$ and $\left(x s+s x^{*}\right)^{2}=S^{2}=0$; we get $0=s\left(x s+s x^{*}\right)^{2}=s(x s)^{2}$, i.e., $s \rho$ is a right ideal nil of bounded index. By Levitski's theorem $s \rho=0$; hence ( $\rho \cap S) \rho=0$. Now, since $R$ has no minimal right ideals, by [3, Lemma 5.1.2.], for $v \notin W$, there exists $x \in \rho$ such that $x^{*} \in \rho, v x^{*}=0$ and $v\left(x+x^{*}\right)=v x \notin W+D v$. But then, by density, there exists $y \in \rho$ such that $v\left(x+x^{*}\right) y \neq 0$, contradicting the fact that $\left(x+x^{*}\right) y \in(\rho \cap S) \rho$ $=0$. This establishes the claim.

Then set $s \in \rho \cap S$ such that $s^{2} \neq 0$. Since $\rho$ is a proper right ideal of $R, s$ is not invertible; moreover, since $\operatorname{dim}_{D} \operatorname{ker} s \geq \operatorname{dim} W>1, d(s)$ $=0$. Hence, by Lemma $4, s d(R) s=0$.

Now, if $x \in \rho$ then $s x^{*}+x s \in \rho \cap S$ and $d(s)=0$ implies $0=$ $d\left(s x^{*}+x s\right)=s d\left(x^{*}\right)+d(x) s$. Since $s d\left(x^{*}\right) s=0$, multiplying by $s$ from the right we get $d(x) s^{2}=0$. Thus $d(\rho) s^{2}=0$. Now, for $x, y \in \rho$, $0=d(x y) s^{2}=d(x) y s^{2}$ forces $d(\rho) \rho s^{2}=0$ and, since $R$ is prime and $s^{2} \neq 0, d(\rho) \rho=0$. Clearly $d(\rho) \neq 0$; so, let $x \in \rho$ be such that $d(x) \neq 0$. If $v d(x) \notin W$ for some $v \in V$, then by density there exists $r \in \rho$ such that $v d(x) r \neq 0$, contradicting the fact that $d(x) r \in d(\rho) \rho=0$. Thus $V d(x) \subset W$ and $d(x)$ is a tranformation of finite rank, a contradiction.

We are now in a position to prove the Theorem:

Proof of the Theorem. By Proposition 1 and Proposition 2 we may assume that $R$ is a simple artinian ring. Hence, $R=D_{n}$, the ring of $n \times n$ matrices over a division ring $D$.

Suppose first that ${ }^{*}$ on $D_{n}$ is of transpose type and assume $n>2$. Let $e_{i j}$ be the usual matrix units. For $i=1, \ldots, n e_{i i}=e_{i i}^{*} \in S$ implies $d\left(e_{l i}\right)=e_{i l} d\left(e_{i i}\right)+d\left(e_{i i}\right) e_{i i}$. Thus, since rank $e_{i i}=1$, rank $d\left(e_{i i}\right) \leq 2$ and, being $n>2, d\left(e_{i i}\right)$ cannot be invertible. Hence $d\left(e_{i l}\right)=0, i=1, \ldots, n$. Thus

Now, if $i \neq j$, for a suitable $0 \neq c \in D, e_{i j}+c e_{j l}=e_{i j}+e_{i j}^{*} \in S$.

$$
\begin{aligned}
d\left(e_{i j}+c e_{j i}\right) & =d\left(e_{i i}\left(e_{i j}+c e_{j i}\right)+\left(e_{i j}+c e_{j i}\right) e_{i l}\right) \\
& =e_{l i} d\left(e_{i j}+c e_{j l}\right)+d\left(e_{i j}+c e_{j l}\right) e_{l i}
\end{aligned}
$$

and so, rank $d\left(e_{i j}+c e_{j l}\right) \leq 2$. It follows $d\left(e_{i j}+c e_{j i}\right)=0$ which implies $0=d\left(e_{i i}\left(e_{i j}+c e_{j i}\right)\right)=d\left(e_{i j}\right)$.

We have proved that $d\left(e_{i j}\right)=0$ for $i, j=1, \ldots, n$. Now let $x \in D$. 
If $i \neq j, \quad S \ni x e_{i j}+\left(x e_{i j}\right)^{*}=x e_{i j}+c_{1} x{ }^{*} c_{2} e_{j i}$ for suitable $c_{1}, c_{2} \in$ $D \cap S$. We have:

$$
\operatorname{rank}\left(d\left(x e_{i j}+c_{1} x{ }^{*} c_{2} e_{j i}\right)\right)=\operatorname{rank}\left(d(x) e_{i j}+d\left(e_{1} x * c_{2}\right) e_{j i}\right) \leq 2,
$$

hence $d\left(x e_{i j}+e_{1} x c_{2} e_{j i}\right)=0$, and, multiplying by $e_{j i}$ from the right we get $d(x) e_{i t}=0$, for all $i=1, \ldots, n$. Thus $d(x)=d(x I)=\Sigma_{i} d(x) e_{i i}=0$, i.e. $d(D)=0$. In short $d=0$ in $D_{n}$.

Now suppose that ${ }^{*}$ is symplectic. In this case $D=F$ is a field and suppose $n>4$. Let $I_{1}=e_{11}+e_{22} ; \quad I_{1}^{2}=I_{1} \in S$, so $\operatorname{rank} d\left(I_{1}\right)=$ $\operatorname{rank}\left(I_{1} d\left(I_{1}\right)+d\left(I_{1}\right) I_{1}\right) \leq 4$ implies $d\left(I_{1}\right)=0$. Now, for $i$ odd, $a=$ $e_{1 i}+e_{i+1,2} \in S$; hence $d(a)=d\left(I_{1} a+a I_{1}\right)=I_{1} d(a)+d(a) I_{1}$ has rank $\leq 4$. It follows $d(a)=0$ and, so, for $i \neq 1,0=d\left(I_{1} a\right)=d\left(e_{1}\right)$. On the other hand, if $i$ is even, $e_{1 i}-e_{i-1,2} \in S$ and by the same argument we get $d\left(e_{1 i}\right)=0$ for $i \neq 2$. Moreover by looking at $e_{1 l}+e_{i 1}^{*}$ as above, we obtain $d\left(e_{i 1}\right)=0$ for $i \neq 1,2$. At this stage it easily follows $d\left(e_{\imath j}\right)=0$ for all $i$, $j=1, \ldots, n$. Since $d\left(I_{1}\right)=0$ implies $d(F)=0$, then $d=0$ in $F_{n}$ and we are done.

We are left with the case $R=F_{4}$ and * symplectic. We will prove that in this case $d$ must be inner. By a well known result on finite dimensional simple algebras it is enough to prove that $d(F)=0$. So, suppose by contradiction that there exists $\alpha \in F$ such that $d(\alpha) \neq 0$ and let $s \in S$, $s \neq 0$, be such that $d(s)=0$. Then, since $d(\alpha) \in F, d(\alpha s)=d(\alpha) s \neq 0$ implying $s$ invertible. Therefore, for every $s \in S, s \neq 0, d(s)=0$ implies $s$ invertible.

Now, if $I$ is the identity matrix in $F_{2}, t=\left(\begin{array}{l}00 \\ 0 I\end{array}\right) \in S$ and, since $t$ is not invertible, $d(t) \neq 0$. Moreover it is easy to prove that $d(t)=\left(\begin{array}{cc}0 & A \\ B & 0\end{array}\right)$ where $A, B \in F_{2}$. Now let $V$ be a 4-dimensional vector space over $F$ and let $\left\{e_{1}\right.$, $\left.e_{2}, e_{3}, e_{4}\right\}$ be the standard basis for $V$. Then since $d(t)$ is invertible, $e_{1} d(t), e_{2} d(t)$ are linearly independent over $F$; moreover $e_{1} d(t), e_{2} d(t)$ $\in \operatorname{Span}_{F}\left\{e_{3}, e_{4}\right\}$.

Clearly, there exists an element $x \in F_{4}$ such that $e_{1} d(t) x=e_{2} d(t) x$ $=0$ and $\operatorname{span}_{F}\left\{e_{1} x, e_{2} x\right\}=\operatorname{span}_{F}\left\{e_{3}, e_{4}\right\}$.Now writing

$$
x=\left(\begin{array}{ll}
X_{11} & X_{12} \\
X_{21} & X_{22}
\end{array}\right)
$$

where $X_{i} \in F_{2}$, we have that $X_{21}$ is a unit and that $\left(t x x^{*} t\right)_{2,2}=X_{21} X_{21}^{*}$ $\neq 0$, a contradiction.

\section{REFERENCES}

[1] J. Bergen, I. N. Herstein and C. Lanski, Derivations with invertible values, Canad. J. Math., 35, 2 (1983), 300-310. 
[2] I. N. Herstein, Topics in Ring Theorey, Univ. of Chicago Press, Chicago, 1969.

[3] __ Rings With Involution, Univ. of Chicago Press, Chicago, 1976.

[4] __ A note on derivations II, Canad. Math. Bull., 22 (1979), 509-511.

[5] _ A theorem on derivations of prime rings with involution, Canad. J. Math., 34, 2 (1982), 356-369.

Received March 6, 1984 and in revised form July 5, 1984. This work was supported by RS $60 \%$ (Italy) and FAPESP (Brazil).

Universita di Palermo

Via ARChINAFi 34

90123 Palermo, Italy

AND

Universidade de São Paulo

Caixa Postal 20.570 Ag. Iguatemi

São Paulo, Brasil 


\section{PACIFIC JOURNAL OF MATHEMATICS EDITORS}

V. S. VARADARAJAN

(Managing Editor)

University of California

Los Angeles, CA 90024

HERBERT CLEMENS

University of Utah

Salt Lake City, UT 84112

R. FINN

Stanford University

Stanford, CA 94305
HERMANN FLASCHKA

University of Arizona

Tucson, AZ 85721

RAMESH A. GANGOLLI

University of Washington

Seattle, WA 98195

VAUGHAN F. R. JONES

University of California

Berkeley, CA 94720

ROBION KIRBY

University of California

Berkeley, CA 94720
C. C. MOORE

University of California

Berkeley, CA 94720

H. SAMELSON

Stanford University

Stanford, CA 94305

HAROLD STARK

University of California, San Diego

La Jolla, CA 92093

\section{ASSOCIATE EDITORS}

R. ARENS

E. F. BECKENBACH

B. H. NEUMANN (1906-1982)
F. WOLF

K. YOSHIDA

\section{SUPPORTING INSTITUTIONS}

UNIVERSITY OF ARIZONA

UNIVERSITY OF BRITISH COLUMBIA

CALIFORNIA INSTITUTE OF TECHNOLOGY

UNIVERSITY OF CALIFORNIA

MONTANA STATE UNIVERSITY

UNIVERSITY OF NEVADA, RENO

NEW MEXICO STATE UNIVERSITY

OREGON STATE UNIVERSITY
UNIVERSITY OF OREGON UNIVERSITY OF SOUTHERN CALIFORNIA

STANFORD UNIVERSITY

UNIVERSITY OF HAWAII

UNIVERSITY OF TOKYO

UNIVERSITY OF UTAH

WASHINGTON STATE UNIVERSITY

UNIVERSITY OF WASHINGTON 


\section{Pacific Journal of Mathematics}

\section{Vol. 123, No. $1 \quad$ March, 1986}

Maria Emilia Alonso García, A note on orderings on algebraic varieties $\ldots \ldots 1$

F. S. De Blasi and Józef Myjak, On continuous approximations for

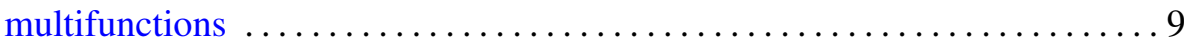

Frank Albert Farris, An intrinsic construction of Fefferman's CR metric . . . 33 Antonio Giambruno, P. Misso and Francisco César Polcino Milies, Derivations with invertible values in rings with involution $\ldots . \ldots . \ldots .47$

Dan Haran and Moshe Jarden, The absolute Galois group of a pseudo real

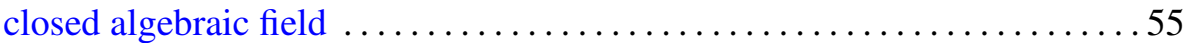

Telemachos E. Hatziafratis, Integral representation formulas on analytic

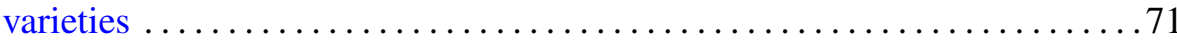

Douglas Austin Hensley, Dirichlet's theorem for the ring of polynomials

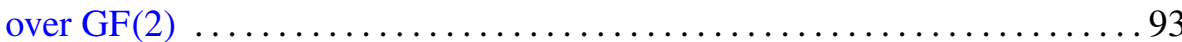

Sofia Kalpazidou, On a problem of Gauss-Kuzmin type for continued

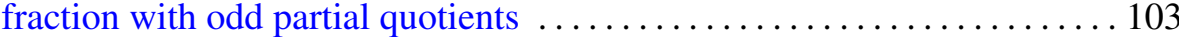

Harvey Bayard Keynes and Mahesh Nerurkar, Ergodicity in affine

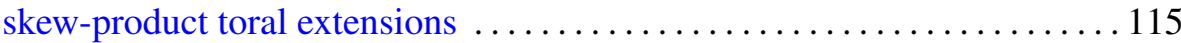

Thomas Landes, Normal structure and the sum-property $\ldots \ldots \ldots \ldots \ldots 127$

Anthony To-Ming Lau and Viktor Losert, Weak*-closed complemented invariant subspaces of $L_{\infty}(G)$ and amenable locally compact groups ...149 Andrew Lelek, Continua of constant distances in span theory . . . . . . . . 161 Dominikus Noll, Sums and products of $B_{r}$ spaces $\ldots \ldots \ldots \ldots \ldots \ldots \ldots \ldots$ Lucimar Nova, Fixed point theorems for some discontinuous operators 189

A. A. S. Perera and Donald Rayl Wilken, On extreme points and support points of the family of starlike functions of order $\alpha$

Massimo A. Picardello, Positive definite functions and $L^{p}$ convolution operators on amalgams ........................

Friedrich Roesler, Squarefree integers in nonlinear sequences ......... 223

Theodore Shifrin, The osculatory behavior of surfaces in $\mathbf{P}^{5}$ 